EPPO Bull. 9 (3): 149-153 (1979)

\title{
Systems Analysis and Dynamic Simulation ${ }^{11}$
}

\author{
by C.T. DE WIT and R. RABBINGE \\ Agricultural University, Wageningen (Netherlands)
}

\section{ABSTRACT}

The state-variable approach to systems analysis and dynamic simulation is based on the assumption that the state of each system at any moment may be quantitatively characterized and that changes in state may be described by mathematical equations.

The basic distinction between state, rate and driving variables in this approach is illustrated by examples.

The conflict resulting from the large number of state variables that may be distinguished in any ecological system and the small number that can be handled in simulation models is treated and attention is given to the distinction between unique and recurring systems, especially with reference to the problem of model evaluation.

\section{Systems and Models}

For more than 30 years, considerable attention has been paid in the engineering sciences to the analysis of complex, dynamic systems, and with considerable success. The approach, which is now being adopted in the biological sciences, is characterized by the terms: systems, models and simulation. A system is a part of reality that contains interrelated elements, a model is a simplified representation of a system, and simulation may be defined as the art of building mathematical models and the study of their properties in reference to those of the system.

Although any model should have definite goals, be lucid and achieve its objective, it seems, in practice, that goals are too often described in such broad terms that sufficient lucidity is reached only for the initiated and that the models are achieving less than expected by the biologists.

\section{State Variable Approach}

A file with data on an ecosystem may be called a model, but is a model without purpose and lucidity. Uses of the data may be formulated and then lucidity may be introduced by statistical treatment of the data. But, the models remain descriptive, showing only the existence of relations between elements, without any explanation, which is, of course, not their purpose to begin with.

However, models that have the purpose of explaining systems are possible in biology, because various levels of organization are distinguished in this science, as in any other natural science. These different levels of organization may be classified,

1) Paper presented at the Joint EPPO/IOBC Conference on Systems Modelling in Modern Crop Protection, Paris, 12-14 October, 1976. 
according to the size of the system and time constant involved, as those of molecules, cell structures, cells, tissues, organs, individuals, populations and ecosystems. Models that are made with the objective of explaining are bridges between levels of organization, which allow the understanding of larger systems with the larger time constants on the basis of the knowledge gained by experimentation on smaller systems with smaller time constants. In this way, the properties of membranes may be understood better by studying molecules, and the properties of ecosystems by studying species.

For models that claim to be of the explanatory type, the state variable approach is gaining wide acceptance. These models are based on the assumption that the state of each system at any moment may be quantitatively characterized and that changes in the state may be described by mathematical equations. This leads to models in which state, rate and driving variables are distinguished.

State variables are quantities like biomass, animal number for a species, the amount of nitrogen in soil, plant or animal, the water content of the soil ; i.e., those variables which can still be measured when time stands still.

Driving variables characterize the interactions at the boundaries of the system, and are continuously measured. Examples are macrometeorological variables, like rain, wind, temperature and radiation, and the food supply or migration of animals over the boundaries of the system. Whether the same variables are driving, state or rate variables depends on these boundaries. For instance, the heat stored within a vegetation canopy is a state variable when the system includes micrometeorological aspects, but a driving variable which has to be measured when the micrometeorological aspects are excluded from the system.

Each state variable is associated with rate variables which characterize their rate of change at a certain instant as a result of specific processes. These variables give the values of flows of material between state variables, for example, between vegetative biomass and a grazing animal. Their value depends on the state and driving variables, according to rules that are based on knowledge of the physical, chemical and biological processes that take place and not on a statistical analysis of the behaviour of the system which is being studied. This is the most important distinction between models that describe and models that attempt to explain.

New values of the state variables are found after calculating all rates; the computing process is usually repeated at given time intervals. In its most elementary form, this is a process of numerical integration and the simulation program may be replaced by an analytical solution in cases where the equations are simple enough, but this is a rare occurrence.

Especially for the uninitiated, attempts are made to simplify simulation programs into relational diagrams, often according to a method that was developed by FORRESTER (1971) to represent models of industrial systems. An example of such a relational diagram is given in figure 1. Here, the hatching of a batch of eggs is given. The number of eggs is represented by a rectangle, a state variable, the hatching rate controls the flow of eggs from one state to another. The flow being represented by a solid arrow, the rate is given by the valve symbol. The dotted lines indicate which state or driving variables affect the rate, without indicating the quantitative aspect: 
these are the flows of information that are considered. In this case, the flow rate of eggs is determined by the number of eggs and the relative rate of development, a temperature-dependent parameter.

Fig. 1

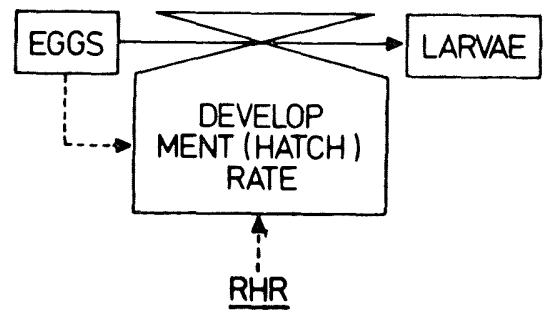

Simplest relational diagam for the hatching process of eggs.

Each rate depends at each moment on state and driving variables only and can, therefore be computed independently of any other rate. Hence, it is never necessary to solve $\mathrm{n}$ equations with $\mathrm{n}$ unknowns. An example may be needed. It is clear that the rate of oviposition of a mite is closely related to the rate of feeding. In a state variable model, this dependency is a result of the simultaneous operation of 2 independent processes. Feeding contributes to the gut content, and this content is one of the states that determine the rate of oviposition. In the absence of food, feeding stops immediately, but oviposition proceeds until the gut is depleted.

\section{Some Problems in Systems Analysìs}

The number of state variables that may be distinguished in an ecosystem are depressingly large. They concern not only primary producers, consumers and decomposers, but also the various species, their number, size, age, sex, stage of development, etc. For plants, not only the weight and surface area of the leaves are of importance, but also their nitrogen and mineral content, their enzymes and other biochemical characteristics. One can continue in this way and, therefore, a model that is based on full knowledge of all biological, physical and chemical phenomena that occur is never realized. Models are simplified representations of systems and the simplification manifests itself by the limited number of state variables that are considered.

By analogy with other approaches, it is assumed that considerable reduction of the number of state variables may be obtained by limiting the boundaries of the model and by focussing interest on those aspects where interest or understanding is most wanted. Then, processes can be ordered with respect to their importance, and only processes within the limited focus need be handled in detail.

The number of state variables that can be considered in any model is very limited, not so much because of the size of the computer or the cost of computer time, but because the research effort that can be invested in any one problem is limited. Models that contain about a hundred state variables are, for this reason, already very large but, at the same time, they may be small compared with the complexity of the ecosystems that are considered. 
For each purpose, there is an optimum in the number of state variables that should be considered. At first, the applicability of the model to the real world problem increases with increasing number of state variables, but then it decreases again, because the addition of new state variables diverts attention from state variables introduced earlier because they were considered more important. The heuristic process of obtaining a set of state variables in order of their importance takes much time and many modelling efforts in ecology are sometimes explicitly, but mostly implicity, geared towards this goal.

\section{Types of Systems}

There are repeatable, recurring and unique systems. Examples of repeatable systems are microbiological (manufacture of vinegar), agricultural (growth of maize), models of pest control, or industrial (manufacture of cars). Examples of recurring systems are stars, individuals of a species and ecological systems with so much resilience that, after disturbance, the original course of development is restored in due course (peat bogs). These recurring ecological systems appear to the observer at different places at the same time, but in different stages. The strength of the field-ecologist lies in his ability to interpret as a time series in one place what is observed in different places at one moment. Repeatable systems can always be analyzed by experimentation, but recurring systems sometimes only by observation. There is, at present, a strong emphasis on the experimental analysis of recurring ecological systems and this is justified because disturbances are dampened and destruction of the system during experimentation may be acceptable because there are many of them.

But, there are also unique ecological systems or ecological systems with unique aspects. These are systems in which development is not governed by negative feedback, so that their development is diverse, although the origin may be the same. Other systems are unique because of the geographical situation, like some estuaries, lakes, islands and, of course, the world as a whole. Models of unique systems are concepts that cannot be validated experimentally, but only more or less verified by observation of the behaviour of the real system over time. They remain, therefore, speculative models. The faith in speculative models is strengthened if similar methods of systems analysis applied to repeatable or recurring systems lead to validated models that cannot be falsified. Such models of physical systems exist: speculative models that predict the chances of flooding on the basis of an analysis of the physical processes are trusted, although sufficient floods for verification never occur within a human lifespan. Whatever the model predicts, the dykes are strengthened as soon as one flood takes place and this proves that trust of this kind has its limits. Speculative models of ecological systems cannot be trusted as yet, because few models that are properly validated exist and the principles of model building in ecology are still being developed. This certainly holds for so-called world models, unless their results are so obvious that the proper conclusions may be drawn without sophisticated techniques.

But, if a speculative model of a unique system is sufficiently trusted, can it be used? For this purpose, it is at least necessary to initialise the model, so that the values of all the state variables have to be determined within such a short time span that they do not change materially. And this should be done without disturbing the unique system to such an extent that its course of development is affected.

In the final analysis, it may appear that the ecologist is in the same position as the outmoded physicist, who claims that it is only necessary to determine at the 
same time the position, mass and velocity of all gas atoms in his room to predict their future. He may be in an even worse position, because he has to live with, or even within, his unique system and cannot escape the problem by using the law of averages.

\section{Model Construction}

In the construction of a dynamic model of repeatable or recurring systems and in its verification and application, different stages can be distinguished. The first step concerns the description of the systems, its limits and its structure; the second step comprises quantification and mathematical description of the rate equations by means of process experiments or literature study (DE WIT and GOUDRIAAN, 1978).

The third step of model building, often closely connected and interwoven with the second step, concerns the construction of computer models according to welldefined techniques. The fourth step is the evaluation and verification of the model (ARNOLD and DE WIT, 1976). This requires independent experiments on the system level. More often than not, the simulated results differ in relevant aspects from these experimental results. Agreement may be obtained by fudging with functions or parameters, but simulation is then degenerated to the most cumbersome method of curve-fitting. Instead, the structure of the model and the basic process experiments should be reconsidered, guided by experimentation with model and real systems.

RESUME

Bull. OEPP 9 (3) : 149-153 (1979)

\section{Analyse de systèmes et simulation dynamique}

par C.T. DE WIT

Agricultural University, Wageningen (Pays-Bas)

L'approche de «variables d'état» à l'analyse de systèmes et à la simulation dynamique se base sur l'hypothèse que l'état d'un système peut être caractérisé de manière quantitative, et que les changements d'état peuvent être représentés par des équations mathématiques.

Cette approche entraîne une distinction fondamentale entre variables d'état, variables de vitesse, et variables forçantes, qui est illustrée d'exemples.

Il existe un conflit entre le grand nombre de variables d'état que l'on peut distinguer dans un système écologique et le faible nombre que l'on peut manipuler dans les modèles de simulation. L'étude souligne ensuite la distinction entre les systèmes uniques et récurrents, notamment en ce qui concerne le problème de l'évaluation des modèles.

\section{REFERENCES}

ArNold, G.W. \& C.T. DE WIT, ed. (1976). Critical evaluation of systems analysis in ecosystems research and management. Simulation Monographs, Pudoc, Wageningen, Netherlands.

Forrester, J.W. (1971). World Dynamics. Wright-Allen Press, Cambridge Mass, USA.

WIT, C.T. DE \& J. GoudriaAn (1978). Simulation of ecological processes. Simulation Monographs, Pudoc, Wageningen, Netherlands. 